\title{
Ionospheric measurements of relative coronal brightness during the total solar eclipses of 11 August, 1999 and 9 July, 1945
}

\author{
C. J. Davis ${ }^{1}$, M. Lockwood ${ }^{1}$, S. A. Bell ${ }^{2}$, J. A. Smith ${ }^{1}$, E. M. Clarke ${ }^{3}$ \\ ${ }^{1}$ Space Science Department, Rutherford Appleton Laboratory, Chilton, OX110QX, OXON, UK \\ ${ }^{2}$ H M Nautical Almanac Office, Rutherford Appleton Laboratory, Chilton, OX11 0QX, OXON, UK \\ ${ }^{3}$ Dept. Science and Mathematics, Sheffield Hallam University, City Campus, Pond Street, Sheffield, S11WB, UK
}

Received: 8 October 1999 / Accepted: 27 October 1999

\begin{abstract}
Swept-frequency (1-10 MHz) ionosonde measurements were made at Helston, Cornwall $\left(50^{\circ} 06^{\prime} \mathrm{N}, 5^{\circ} 18^{\prime} \mathrm{W}\right)$ during the total solar eclipse on August 11, 1999. Soundings were made every three minutes. We present a method for estimating the percentage of the ionising solar radiation which remains unobscured at any time during the eclipse by comparing the variation of the ionospheric E-layer with the behaviour of the layer during a control day. Application to the ionosonde date for 11 August, 1999, shows that the flux of solar ionising radiation fell to a minimum of $25 \pm 2 \%$ of the value before and after the eclipse. For comparison, the same technique was also applied to measurements made during the total solar eclipse of 9 July, 1945 , at Sörmjöle $\left(63^{\circ} 68^{\prime} \mathrm{N}, 20^{\circ} 20^{\prime} \mathrm{E}\right)$ and yielded a corresponding minimum of $16 \pm 2 \%$. Therefore the method can detect variations in the fraction of solar emissions that originate from the unobscured corona and chromosphere. We discuss the differences between these two eclipses in terms of the nature of the eclipse, short-term fluctuations, the sunspot cycle and the recently-discovered long-term change in the coronal magnetic field.
\end{abstract}

Key words: Ionosphere (solar radiation and cosmic ray effects) - Radio science (ionospheric physics) Solar physics, astrophysics, and astronomy (corona and transition region)

\section{Introduction}

Radio measurements of the Earth's ionosphere during total solar eclipses are not new, the first recorded being in 1912 by W. H. Eccles (Eccles, 1912); however, the

Correspondence to: C. J. Davis reasons for making such measurements have changed. Initial interest centred on the effect of eclipses on the propagation of radio waves. The eclipse of 31 August, 1932, was studied intensively for changes in the ionosphere (e.g. Mimno and Wang, 1933). It was through measurements such as these that it was possible to show that the ionosphere was predominantly generated by electromagnetic radiation from the Sun, rather than by particles in the solar wind. Eclipse measurements then focused on the ionospheric continuity equation, in an effort to determine the coefficients determining the electron loss rate. For the E-region, it was shown that photochemical processes dominated over transport and that the continuity equation took the form:

$\frac{\mathrm{d} N}{\mathrm{~d} t}=q_{\mathrm{o}} f(\chi)-\alpha N^{2}$

where $N$ is the electron concentration, $q_{\mathrm{o}}$ is the production rate for an overhead Sun, $\chi$ is the solar zenith angle and $\alpha$ is the ionospheric loss rate coefficient. The function $f(\chi)$ is $\cos (\chi)$ for a flat, horizontally stratified atmosphere but is the more complex Chapman function, $C h(\chi)$, when allowance is made for a curved Earth. Much effort was expended trying to obtain the value of $\alpha$. During an eclipse, it was reasoned, all radiation from the Sun would be gradually obscured until the production rate became zero at totality. Equation (1) was therefore rewritten in the form;

$\frac{\mathrm{d} N}{\mathrm{~d} t}=A q_{\mathrm{o}} f(\chi)-\alpha N^{2}$

where $A$ is the fraction of the exposed photospheric disk. At totality, $A=0$ so $\alpha$ was estimated from Eq. (2) to be:

$\alpha=-\frac{\mathrm{d} N}{\mathrm{~d} t} / N^{2}$

Although many eclipses were used to estimate $\alpha$ in this way, the results were very variable and were significantly less than the values predicted by theory and obtained by other observation methods. It was eventually concluded that the reason for this was that there was, in fact, a 
significant amount of ionising radiation coming from regions in the solar atmosphere above the eclipsed photosphere. Some researchers then went on to estimate a value for the fraction of the radiation coming from the solar atmosphere (e.g. Minnis, 1954) by adopting a theoretical value for $\alpha$.

Although the quest for a value of $\alpha$ from eclipse experiments ultimately proved unsuccessful, these historical data sets provide extremely useful information about the ionising radiation produced by the solar corona and chromosphere. In this work we present a technique aimed at exploiting these data.

Recent work (Lockwood et al., 1999a, b; Stamper et al., 1999) has revealed long-term changes occurring in the solar corona. Measurements of the fluctuation level in the Earth's magnetic field made since 1868 (the $a a$ index) show the eleven year solar cycle variation superimposed on an increasing long-term trend. By comparing these measurements with in-situ satellite measurements of the Interplanetary Magnetic Field (IMF) made since 1968, Stamper et al. (1999) were able to show that the long-term upward drift in $a a$ was due to an increase in the IMF around the Earth. The IMF was also shown to obey Parker spiral theory on average, so that the mean radial field at the Earth can be related to the field leaving the Sun through the "coronal source surface".

Measurements from the Ulysses spacecraft have shown that the magnetic coronal source field is, to a good approximation, uniform for all solar latitudes (Balogh et al., 1998; Lockwood et al., 1999b). Consequently, Lockwood et al. (1999a, b) were able to use values of the IMF inferred from the $a a$ data to estimate the total coronal source flux since 1868 . The method was tested by Lockwood and Stamper (1999) and shown to be accurate to a few percent. The results show that the total coronal source flux has more than doubled during this century. The coronal magnetic field is thought to be the source of energy that heats the corona, thereby making it emit strongly at the extreme ultra-violet (EUV) and X-ray wavelengths that ionise the Earth's atmosphere. Therefore, we would expect higher fluxes of ionising radiations from the corona when the coronal source flux is higher. During an eclipse, the limb corona and some of the limb chromosphere remain unobscured at totality, when the photosphere is fully obscured. (Note that the chromosphere and corona close to the Sun-Earth axis will, like the photosphere, be obscured by the Moon). From this, a large coronal source magnetic flux is expected to be associated with a higher fraction of the total ionising radiation that remains unobscured during totality.

The main interest in this eclipse was therefore to make measurements of the E-region decay and compare these with historical eclipses in an attempt to determine whether the percentage of ionising radiation emitted by the chromosphere and corona has increased over the last century. We concentrate our efforts here on developing the method and testing it by comparing the eclipse of 11 August, 1999, with a very similar eclipse which occurred over Canada and Scandinavia on 9 July, 1945. These eclipses occurred at similar times of year and at similar points of the 11-year solar activity cycle. A detailed survey of many other eclipse measurements will be made in a future paper.

\section{Method}

In the current study, we adopt a similar approach to the previous work by defining a function, $\Phi$, which represents the unobscured fraction of the total solar ionising radiation at a given time during an eclipse. This differs from the previous approach in that there is no assumption that the ionising radiation is coming from the solar disk. Thus the factor $A$ in Eq. (2) is replaced by $\Phi$, giving

$\frac{\mathrm{d} N_{E}}{\mathrm{~d} t}=\Phi q_{\mathrm{o}} f(\chi)-\alpha_{E} N_{E}^{2}$

where the subscript $E$ refers to data taken on the eclipse day. In contrast, the data that would have been recorded on the same day, had the eclipse not occurred, would have obeyed Eq. (1). We describe how we derive a "control day" which is our best estimate of this hypothetical day (that is identical to the eclipse day, other than the Moon does not obscure the Sun). The values of $q_{0}$ and $f(\chi)$ at any one time are the same on the control day and the eclipse day, thus combining Eqs. (1) and (4) gives the following expression for the factor $\Phi$;

$\Phi=\frac{\left[\frac{\mathrm{d} N_{E}}{\mathrm{~d} t}+\alpha N_{E}^{2}\right]}{\left[\frac{\mathrm{d} N_{C}}{\mathrm{~d} t}+\alpha N_{C}^{2}\right]}$

where the subscript $C$ refers to data from the control day. Equation (5) assumes that $\alpha$ is the same on the eclipse and control days $\left(\alpha=\alpha_{E}=\alpha_{C}\right)$. This assumption is analysed in the next section.

\section{Effects of uncertainties in the value of $\alpha$}

If the electron density and its rate of change during both the eclipse and control days are well defined, the only remaining uncertainty in determining $\Phi$ lies in the value of $\alpha$. To a good approximation, $\alpha$ can be represented by the rate coefficient of the reaction;

$\mathrm{O}_{2}^{+}+e \rightarrow O\left({ }^{1} D\right)+O\left({ }^{3} P\right)+5 e v$

The rate coefficient for this reaction is, $\alpha=1.9 \times$ $10^{-10}\left(T_{e} / 300\right)^{-0.5} \mathrm{~m}^{3} \mathrm{~s}^{-1}$ (Rees, 1989), where $T_{e}$ is the electron temperature (which equals the neutral gas temperature at E-region altitudes). It was found that altering the value of $\alpha$ in Eq. (5) by even an order of magnitude had negligible effect on the calculated value of $\Phi$. In order to be confident that the uncertainty in $\alpha$ would not exceed this, we used the MSIS model of the neutral atmosphere (Hedin, 1987) to estimate the temperature for a range of possible geomagnetic activities and exospheric temperatures. An estimate of the temperature at $100 \mathrm{~km}$ (close to the height of the $\mathrm{E}$ region peak, as observed during the 1999 eclipse) was 
obtained by running the model for the relevant geomagnetic and solar conditions (quantified by the Ap index, sunspot number and $10.7 \mathrm{~cm}$ solar flux). This temperature proved insensitive to a range of values of Ap. A change in temperature of $2 \%$ was predicted per increment of $\mathrm{Ap}$, giving only a $1 \%$ change in $\alpha$.

An error may arise from the assumption that the $\alpha$ on our eclipse day and on the control day are the same, given that the atmosphere will be cooled by the eclipse. Global modelling of the August 11, 1999, eclipse (Müller-Wodarg, 1998) predicted a drop in exospheric temperature of the order of $40 \mathrm{~K}$ under the eclipse path. When such a temperature drop was used with MSIS86, the variation in temperature at $\mathrm{E}$ region altitudes was less that $1 \mathrm{~K}$ which, from the information given already, gives a negligible change in $\alpha(\approx 0.5 \%)$. Thus we can use $\alpha_{E}=\alpha_{C}$ to a high degree of accuracy.

Lastly, our control day is based on day(s) close to the actual eclipse day. Since the neutral atmosphere has a large thermal capacity, however, the temperature on consecutive days is similar and we can allow for small day-to-day variations by adjusting the control day variation in $N_{C}$ to provide a good fit to the eclipse day data $N_{E}$ outside the eclipse period itself (see later). From these considerations, we conclude that the random and systematic uncertainties in $\alpha$ are of the order of $1 \%$, well within the range we require to make $\Phi$ insensitive to the uncertainties in $\alpha$.

\section{Measurements during the eclipse of 11 August, 1999}

A Lowell DPS 1 digital ionosonde (Bibl and Reinisch, 1978), operated by the ionospheric monitoring group of the Rutherford Appleton Laboratory, was deployed under the path of totality during this eclipse, on the cricket pitch of the Lower School in Helston, Cornwall $\left(50^{\circ} 6^{\prime} \mathrm{N}, 5^{\circ} 18^{\prime} \mathrm{W}\right)$. Figure 1 shows the zone of totality over the UK mainland at the E-region height of $100 \mathrm{~km}$.
It can be seen from this figure that Helston lay on the extreme northern limit of totality in the E-region. The centre line of the eclipse at this altitude lay approximately $50 \mathrm{~km}$ south of the centre line at sea-level.

The ionosonde was set up to transmit frequencies between 1 and $10 \mathrm{MHz}$ in $50 \mathrm{kHz}$ steps. Each of these frequency sweeps took around one minute. In between such sequences, the sounder carried out detailed measurements at a limited set of frequencies relevant to the F region. These "sky map" measurements will be discussed in a future publication. The sounder repeated this sequence every three minutes.

The data were automatically scaled and then checked manually. Where the $\mathrm{E}$ region critical frequency, $f_{\mathrm{oE}} \mathrm{E}$, was well defined, a value could be given to an accuracy of $\pm 0.1 \mathrm{MHz}$. Under circumstances where blanketing by sporadic $\mathrm{E}$ echoes occurred, $f_{\mathrm{OE}}$ was defined by any cusp seen in the E layer trace. The accuracy of $f_{\mathrm{oE}}$ was then taken to be half the difference between this value and the lowest $\mathrm{F}$ region echo, fmin. If the start-time of the sounding is known, the time at which $f_{\mathrm{oE}}$ is measured can be determined by its position in the sequence. Due to processing time at the beginning of each sequence however, the uncertainty in the time of the $f_{\mathrm{oE}}$ measurement can only be quoted to $\pm 5 \mathrm{~s}$.

Since this eclipse also coincided with the Perseid meteor shower, some of the data surrounding the eclipse day was blanketed by dense sporadic E layers $\left(E_{\mathrm{s}}\right)$ formed from the meteor trails at altitudes of around $100 \mathrm{~km}$.

Values of $f_{\mathrm{oE}}$ were obtained throughout the day before (10 August) which we adopted as the basis for our control day. In this investigation, we are not interested in rapid variations in the layers caused by transient phenomena such as waves or by transient solar events like flares on the control day. For this reason, a curve was fitted to the control day data in order to remove any noise from our calculation of the gradients on this day. The uncertainty in the density measurements on the control day was then estimated from the

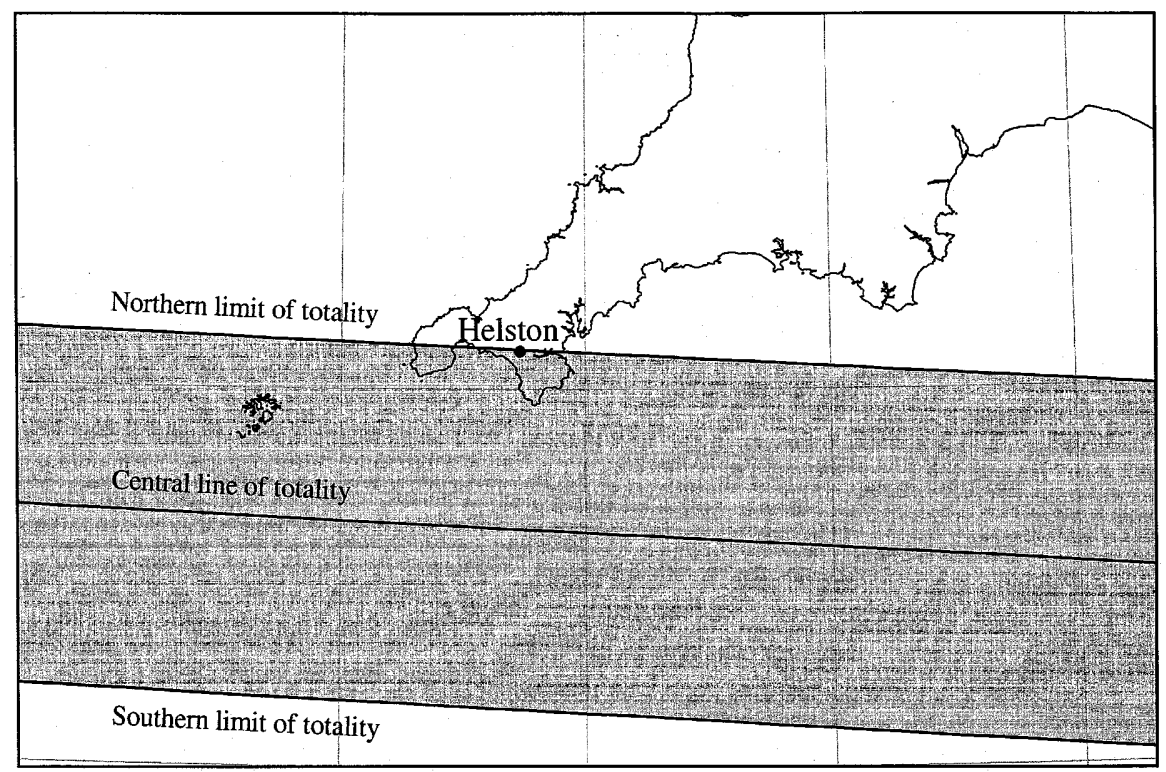

Fig. 1. The zone of totality at an altitude of $100 \mathrm{~km}$ across the southwest tip of the UK during the eclipse of August 11, 1999. The observation point at Helston lay on the extreme northern limit of this zone where totality lasted $8 \mathrm{~s}$ 
variation of the data about this curve. Although past eclipse experiments have taken the control day to be an average of a range of days prior to the eclipse, some of these have highlighted the inadequacies of this approach (e.g. Minnis, 1954). Thus we elected to compare the eclipse day with a control day based on the day before the eclipse wherever possible. This has the effect of minimising any systematic errors introduced by a variation in $q_{\mathrm{o}}$ or $\alpha$ resulting from changes in solar conditions. Once the shape of the control day $f_{\mathrm{oE}}$ variation has been established in this way, the effect of any day-to-day variation in $q_{0}$ and $\alpha$ is further minimised by scaling the control day variation in peak density $N_{C}(t)$ to match the peak density variation, $N_{E}(t)$, on the eclipse day prior to the eclipse itself. It was found that the control data required scaling by a factor of 0.9 to bring it in line with the ionospheric variation on the morning of the eclipse. The necessity of scaling the control data in this way can be illustrated by studying the variation in midday $f_{\mathrm{oE}}$ at Chilton $\left(51^{\circ} 42^{\prime} \mathrm{N}\right.$, $1^{\circ} 20^{\prime} \mathrm{W}$ ) for the month of August 1999. For this period,

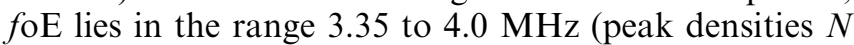
in the range $1.38 \times 10^{11} \mathrm{~m}^{-3}$ to $1.98 \times 10^{11} \mathrm{~m}^{-3}$ ) and so scaling factors between about 1.20 and 0.8 apply. Once scaled, the diurnal variations $N(t)$ were the same, to within the uncertainties that we estimate for our control day. This uncertainty level results from changes in $q_{\mathrm{o}}$ caused by variations in the intensity of solar ionising radiation over time scales of less than one day.

Helston was near the extreme northern limit of the zone of totality in the E-region, with $100 \%$ of the photosphere covered at maximum eclipse for $8 \mathrm{~s}$. First contact at $100 \mathrm{~km}$ occurred at 08:55:36 UT, with totality between 10:10:53 and 10:11:01 UT and fourth contact occurred at 11:31:26 UT. Figure 2 shows the variation of peak electron density for both 10 August and the eclipse day. Good clean ionograms with clear $\mathrm{E}$ region virtual heights $\left(h^{\prime} \mathrm{E}\right)$ and critical frequencies $(f \mathrm{OE})$ were recorded throughout the eclipse, including the critical period surrounding the time of maximum obscuration. Between about 12 UT and 16 UT, sporadic-E formed, reducing the accuracy of these measurements. Nevertheless, a well-defined drop in electron density can be seen prior to this time. The E layer peak was at a height of around $105 \pm 5 \mathrm{~km}$ throughout both days. Figure 2 also shows the polynomial fit to the 10 August data.

Figure $3 \mathrm{a}$ shows the density variation observed during and around eclipse $N_{E}(t)$, plus our "control day" variation, $N_{C}(t)$. The latter is the fitted polynomial shown in Fig. 2, scaled by a factor 0.9 to give the best least-squares fit to the data prior to the first contact (08:55:36 UT). In effect, we have used the shape of the polynomial fit to interpolate the eclipse day data into the eclipse period. The fact that after the eclipse the values return to our control day values gives us confidence that we have a valid control day variation (i.e. the variation that would have been seen that day in the absence of the eclipse) with no systematic errors introduced by day-today variations. Although such scaling may seem unnecessary in this example, other historic eclipses (e.g. Minnis, 1954) show a clear difference between the control and eclipse days. Such control data would certainly require scaling by the current method. Where enough data exists prior to the onset of an eclipse we therefore recommend that the control data be scaled as outlined earlier. Figure $3 \mathrm{~b}$ shows the variation of $\Phi$ for the same period as Fig. 3a, derived using Eq. (5). Uncertainties have been computed carefully, allowing for errors in all terms in Eq. (5). They are large where $N_{E}$ is large but as the difference between $N_{C}$ and $N_{E}$ grows,

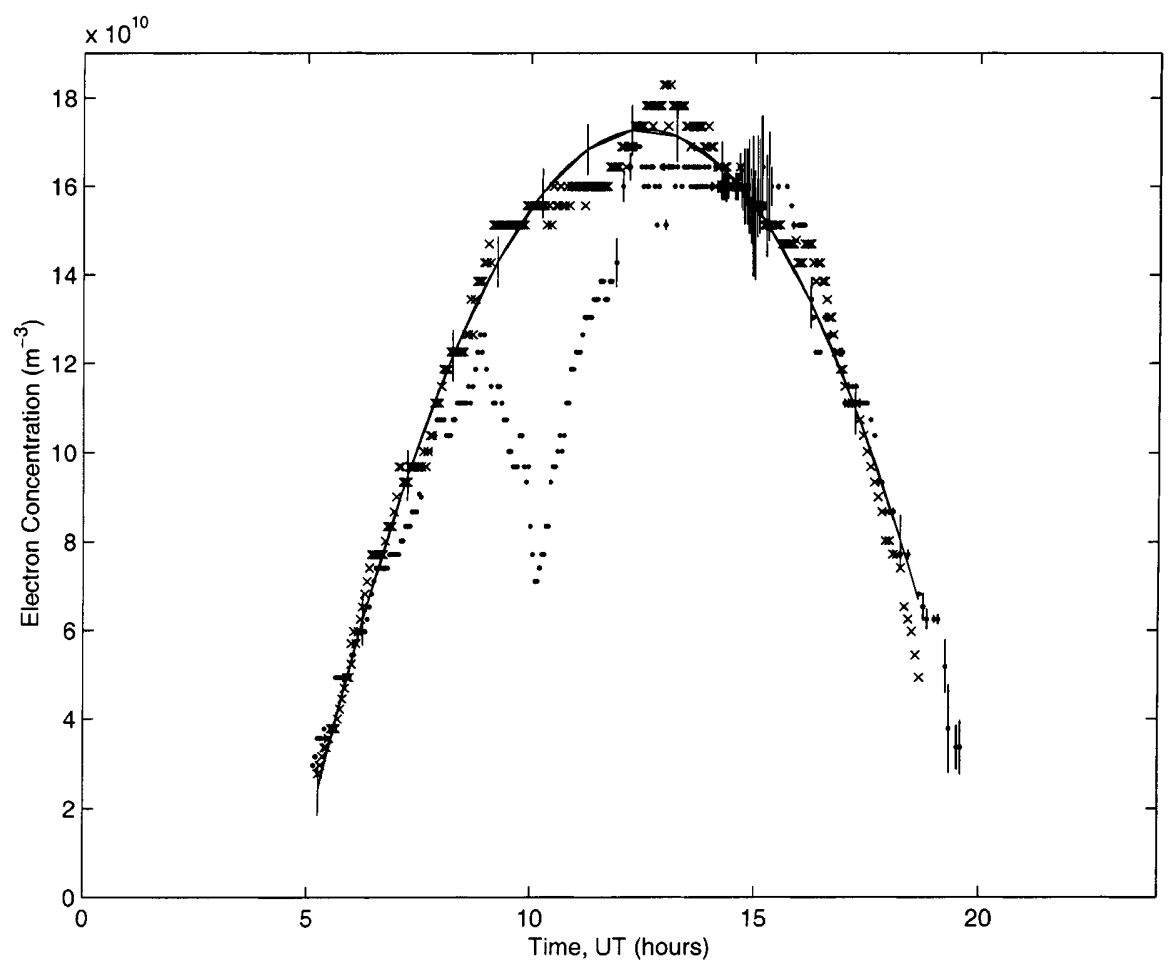

Fig. 2. Electron concentration measurements for 11 August (dots), 10 August, 1999 (crosses), and a polynomial fit to the data from 10 August, 1999 (solid line). The "control day" is obtained from the fitted polynomial and scaled by a constant multiplying factor to give the best leastsquares fit to the data, prior to first contact on 11 August. Note that the polynomial fit shown in this figure has not yet been scaled to fit the eclipse day 

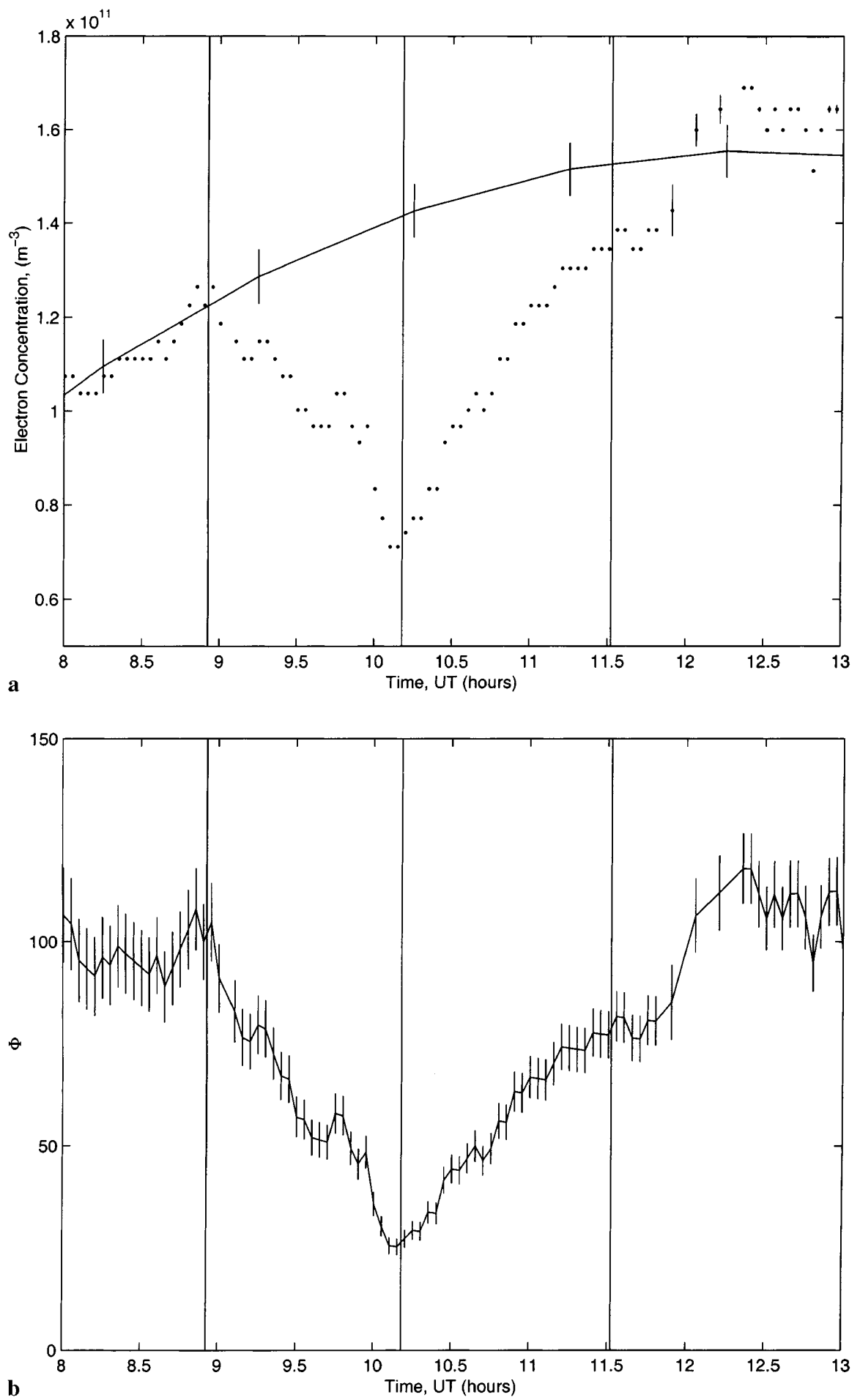

Fig. 3. a Electron concentration measurements along with the scaled "control day" curve for the duration of the 1999 eclipse. Note that in this figure, the control day data have now been scaled by a factor of 0.9 to best match the data prior to the eclipse. The three vertical lines indicate the times of first, second and fourth contact at $100 \mathrm{~km}$ with respect to the photosphere. b The obscuration function, $\Phi$ (in \%) for the duration of the 1999 eclipse. $\Phi$ reaches a minimum value of $25 \pm 2 \%$ the uncertainties in $\Phi$ become smaller. At the time of maximum obscuration of ionising radiations, $\Phi$ falls to a minimum value of $\Phi_{\min }=25 \pm 2 \%$.

\section{Measurements during the eclipse of 9 July, 1945}

The path of totality for the eclipse of 9 July, 1945, passed over both Canada and Sweden and measurements of the ionospheric $\mathrm{E}$ region were made at locations in these two countries. For this study we use data from Sörmjöle, Sweden $\left(63^{\circ} 68^{\prime} \mathrm{N}, 20^{\circ} 06^{\prime} \mathrm{E}\right)$. At this location, the eclipse was total at E region altitudes (Rydbeck, 1946) as shown in Fig. 4. Although these sounders recorded the ionograms on photographic film, the critical frequencies were still accurate to within $\pm 0.1 \mathrm{MHz}$ while the uncertainty in the timing was of the order of $\pm 30 \mathrm{~s}$.

With data from previous eclipse campaigns, we are often limited to the information provided in publications as the raw data have been discarded from archives. 


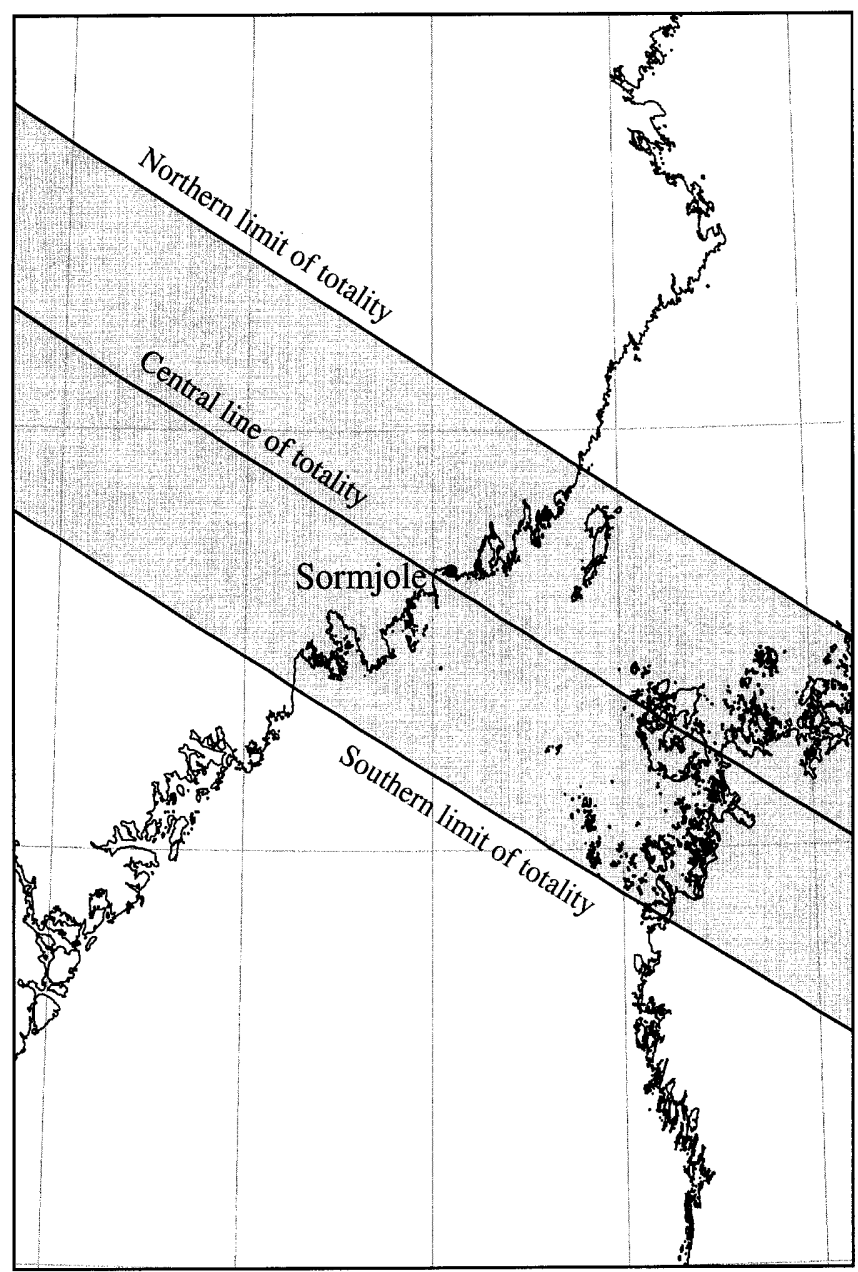

Fig. 4. The zone of totality at an altitude of $100 \mathrm{~km}$ across Sweden in the region of the observation point at Sörmjöle during the eclipse of 9 July, 1945. Sörmjöle lay very near the centre line, where totality lasted $1 \mathrm{~min} 11 \mathrm{~s}$

Fortunately, all of the information required by our method can either be recreated (such as the path of totality) or gleaned from the publications (such as the variations in electron concentration). The only significant difference between the modern and historic measurements is the method of determining the control data. For Sörmjöle, the quoted control data is the average of several days prior to the eclipse. Averaging the data in this way will have the result of reducing the uncertainties at any one time due to transient events, but risks introducing further uncertainties if the value of $q_{\mathrm{o}}$ or $\alpha$ vary within this period. Averaging for carefully selected days was required for the Sörmjöle data because the higher latitudes meant that production by variable auroral precipitation was a factor.

Geomagnetic conditions at Sörmjöle were active, with an ionospheric storm commencing on 6 July (Rydbeck, 1946). This had the effect of disturbing the $F$ layer ionisation but no effect on the $E$ layer is reported. There were no serious disturbances noted between 6 and 9, July. Measurements were taken from June 20, 1945, to July 19, 1945. It is unclear which of these data are used to construct the control data for this experiment. Figure 5a shows the variation of electron concentration for the averaged control day $N_{C}(t)$ and the eclipse day variation $N_{E}(t)$. As for the 1999 eclipse, there is a marked drop in electron concentration on the eclipse day in comparison with the control data and the control day procedure has given very good agreement prior to first contact and generally good agreement to the more smoothed data after recovery from the eclipse perturbation. Thus we conclude that the differences between the two methods for determining the control day are not significant. This is important because in many cases the raw $f$ oE data used to construct the control day are no longer available to us. Figure $5 \mathrm{~b}$ shows the calculation of the obscuration factor $\Phi$ for this period: the minimum value, $\Phi_{\min }$ is $16 \pm 2 \%$.

\section{Discussion and conclusions}

This study has demonstrated a method for determining the percentage of solar ionising radiation which is emitted from the solar atmosphere (the chromosphere and the corona). A comparison has been made between two eclipses occurring at similar points on the solar cycle and at similar times of year. It appears from this comparison that the percentage of solar ionising radiation emitted from the solar atmosphere was greater in the more recent eclipse. This difference was significantly greater than the uncertainties. For the 1945 eclipse, the value of $\Phi_{\min }$ is lower and there is no detectable decrease in $\Phi$ from unity before first contact nor after fourth contact (see Fig. 5b). Thus at times outside any obscuration of the photosphere, we can detect no effect of the $\operatorname{limb}$ corona being obscured. In Fig. $3 b$ on the other hand, there is clear evidence that $\Phi$ is less than 1 outside the times of photospheric obscuration, particularly after the fourth contact at E-region heights. This implies a bright patch of corona is obscured by the Moon at this time, consistent with the higher $\Phi_{\min }$. The differences in $\Phi$ between prior to first contact and after fourth contact imply a structured corona. Using data from other ionosonde sites we will map this structure in a later publication. There are a number of potential causes of the differences in $\Phi_{\min }$. These are discussed in the following subsections.

\subsection{Layer tilts and off-vertical reflections}

Figures 1 and 4 show that Helston is much closer to the edge of the path of E-region totality in the 1999 event than Sörmjöle was in 1945. This means that Helston is more likely to have been detecting off-vertical echoes from the north, east or west of the sounder where the ionosphere is less obscured, and hence slightly denser. The effects of such "tilts" in the E-layer could be detected by direction-of-arrival information of echoes. However, this is not available for past campaigns and so we do not wish to use it to resolve this possibility. However, in many campaigns data were recorded at a 

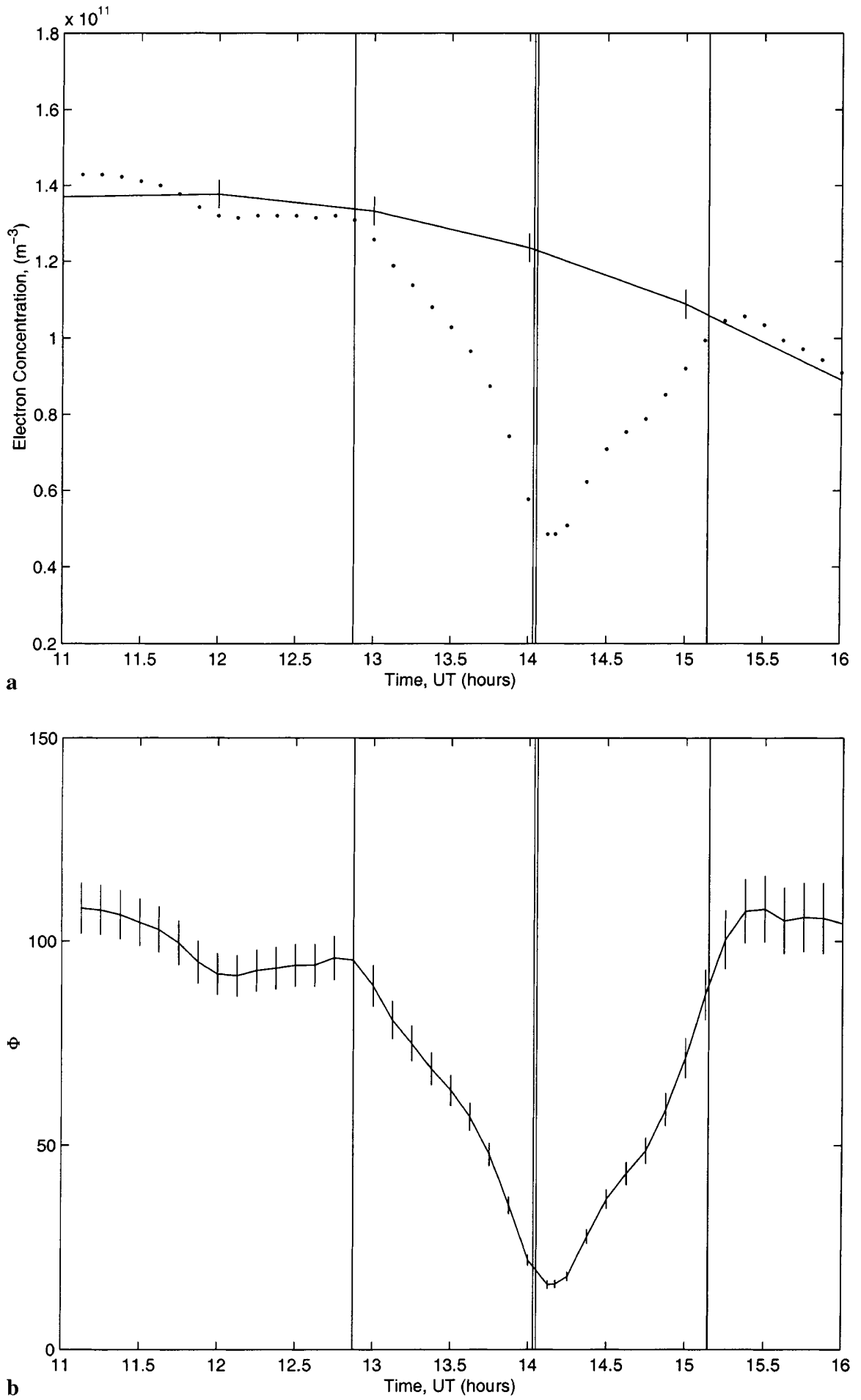

Fig. 5. a Electron concentration measurements along with the "control day" curve for the duration of the 1945 eclipse. The four vertical lines represent first, second, third and fourth contact at $100 \mathrm{~km}$ with respect to the photosphere. $\mathbf{b}$ The obscuration function, $\Phi$ (in \%) for the duration of the 1945 eclipse. $\Phi$ reaches a minimum value of $16 \pm 2 \%$ variety of sites, as for the 1999 event. Application of our method to other sites shows that $\Phi_{\min }$ does not increase with distance from the path totality in the E-layer in a simple manner, which indicates that the coronal structure that is evident in the Helston data is a bigger factor than off-vertical echoes.

We can estimate the effect of layer tilts and the offvertical echoes that they produce. In both eclipses studied here, the peak rates of change in the observed E-layer peak density is seen shortly before and after totality, when $\left|\mathrm{d} N_{E} / \mathrm{d} t\right| \approx 0.33 \mathrm{~m}^{-3} \mathrm{~s}^{-1}$. The eclipse features sweep over the ionosphere at $V_{E}=\mathrm{d} x / \mathrm{d} t \approx$ $0.9 \mathrm{~km} \mathrm{~s}^{-1}$, which means that spatial gradients in peak density are $\mathrm{d} N_{E} / \mathrm{d} x \approx 4 \times 10^{4} \mathrm{~m}^{-4}$. The sounder antenna can detect echoes at angles up to about $30^{\circ}$ from the vertical but E-region echoes are usually within a smaller angle of vertical $\chi_{E}$. We will consider the effect of adopting different values for $\chi_{E}$ below, but use a typical upper limit for $\chi_{E}$ of $15^{\circ}$ in the first instance. This means that E-region echoes are received up to $\Delta x \approx 27 \mathrm{~km}$ 
from directly overhead. With the gradient $\mathrm{d} N_{E} / \mathrm{d} x$ mentioned this gives a difference of densities from the centre to the edge of the field-of-view of $\Delta N_{E} \approx 1 \times$ $10^{9} \mathrm{~m}^{-3}$. If we take a simple parabolic model of the Eregion ionisation profile, with layer semi- thickness $y$ of $15 \mathrm{~km}$, the tilt angle of the iso-density contours in the E-region will be $\varepsilon=\tan ^{-1}\left\{\left(\Delta N_{E} / N_{E}\right)^{\mathrm{T} / 2}(y / \Delta x)\right\} \approx 3.8^{\circ}$. Thus the ray path will be shifted by about $3.8^{\circ}$ from the vertical. In this case the reflection point is $7 \mathrm{~km}$ from overhead and the $N_{E}$ observed would be higher than the overhead values by just $2.5 \times 10^{8} \mathrm{~m}^{-3}$. This is smaller than the measurement uncertainty, set by the frequency resolution of the sounder, which is $\pm 6 \times 10^{8} \mathrm{~m}^{-3}$.

This analysis was based on an angular field of view of $\chi_{E}=15^{\circ}$. It turns out that this value yields the maximum layer tilt angle $\varepsilon$ and thus the maximum shift in the observed $N_{E}$. This is because at smaller $\chi_{E}$, the difference $\Delta N_{E}$ becomes smaller; whereas at larger $\chi_{E}$, $\Delta N_{E}$ is larger, but the shape of the E-layer profile means that the tilt angle $\varepsilon$ is actually reduced. Thus we can conclude that tilt effects in the E-region are not significant and can be neglected.

\subsection{The chromosphere}

Eclipses range from total (in which all of the photosphere is obscured) to annular (in which a ring of photosphere remains unobscured). Similarly in total eclipses with respect to the photosphere, the thin chromospheric layer remains unobscured to varying extents. This depends on the ratio of the Sun-Earth and Moon-Earth distances and would also vary with any change in solar radius. This is important because the chromosphere emits strongly at the wavelengths that ionise the upper atmosphere. Detailed calculation of each case is complex and must include the detailed limb profile of the Moon.

\subsection{Solar activity variations}

The ionising radiations emitted by the Sun and its atmosphere vary on a variety of time scales between day-to-day fluctuations and the solar cycle oscillation. There may well also be longer-term cycles and drifts.

Therefore, the Sun may have been, by chance, more active (in terms of faculae and flares) on the day of the 1999 eclipse than it was on other days around that day. However, this chance is unlikely to be repeated consistently in a study of a great many events.

The difference in $\Phi_{\min }$ values could also reflect the difference in sunspot activity on these two days. Both days are in the rising phase of the solar cycle but the 1945 event was for a daily sunspot number, of 29, whereas provisional values for the 1999 event are 61 and 79 (from Meudon and Boulder, respectively). However it is also possible that the difference reflects a difference in coronal source flux, $F_{s}$. This flux shows a strong solar cycle variation, but superposed on a long-term drift. Lockwood et al. (1999a) and Lockwood and Stamper
(1999) have provided a procedure that enables annual means of $F_{s}$ to be computed. Unfortunately, at the time of writing, values of $F_{S}$ only up to 1996 can be estimated. However, Lockwood et al. (submitted 1999c) have shown that the minimum value of $F_{s}$, just after each sunspot minimum, is a very good predictor of values later in the cycle. The prior minima in $F_{S}$ are $3.21 \times 10^{14} \mathrm{~Wb}$ and $3.29 \times 10^{14} \mathrm{~Wb}$ for the 1945 and 1999 eclipses, respectively. From these, the regression of the kind shown by Lockwood et al. (1999c) yield $F_{S}$ values of $4.71 \times 10^{14} \mathrm{~Wb}$ and $4.79 \times 10^{14} \mathrm{~Wb}$ respectively. Thus $F_{s}$ was, like the sunspot number, $R$, higher for the 1999 event than the 1945 event. By studying all ionosonde observations of eclipses this way, we hope to determine if the $\Phi_{\min }$ values correlate best with $R$ or with $F_{s}$ the key differences being most well-defined at sunspot minimum where the behaviour of $F_{s}$ and $R$ are quite different (Lockwood et al., 1999a).

We have presented a method here, which enables us to determine variations in the fraction of ionising solar radiations that originate in the $\operatorname{limb}$ corona and chromosphere. There are a great number of eclipse observations to which the method can be applied. Eclipses are snapshots of the Sun's condition and thus our estimates will be subject to short time scale fluctuations as well as long-term variations like solar cycle changes and longer term drifts. Once we have found data for past eclipse campaigns and subjected them to this analysis, we will cross correlate with smoothed sunspot number and coronal source flux estimates. In both cases, any short-term variations will cause scatter but should not hide a trend. A strong correlation of $\Phi_{\min }$ with $F_{s}$ would confirm that coronal brightness changes are linked to the energy available for coronal heating, stored in the coronal magnetic field. If found, long-term drifts in both $F_{S}$ and $\Phi_{\min }$ would strengthen the conclusion of Lockwood and Stamper (1999) and Lockwood et al. (1999b) that the coronal source field drift is associated with significant brightness changes and would quantify the fraction of these that are at EUV and X-ray wavelengths.

Acknowledgements. This work was funded by the UK Particle Physics and Astronomy Research Council. We also thank the Radiocommunications Agency for financial assistance with the 1999 observing campaign and the World Data Centre C1 for Solar Terrestrial Physics for supplying the geomagnetic and solar indices.

The Editor-in-chief thanks E. Antonucci for her help in evaluating this paper.

\section{References}

Balogh, A., E. J. Smith, B. T. Tsurutani, D. J. Southwood, R. J. Forsyth, and T. S. Horbury, The heliospheric field over the south polar region of the Sun, Science, 268, 1007-1010, 1995.

Bibl, K., and B. W. Reinisch, The university digital ionosonde, Radio Sci., 13(3), 519-530, 1978.

Eccles, W. H., Effect of the eclipse on wireless telegraphic signals., Electrician, 69, 109, 1912.

Hedin, A. E., MSIS-86 thermospheric model, J. Geophys. Res., 92, 4649-4662, 1987. 
Lockwood, M., and R. Stamper, Long-term drift of the coronal source magnetic flux and the total solar irradiance, Geophys. Res. Lett., 26, 2461-2464, 1999.

Lockwood, M., R. Stamper, and M. N. Wild, A doubling of the Suns' coronal magnetic field during this century, Nature, 399, 6735, 437-439, 1999a.

Lockwood, M., R. Stamper, and M. N. Wild, The implications of the long-term variation of the coronal source flux for predictions of the amplitude of the solar cycle. Annales Geophys, in press, 1999c.

Lockwood, M., R. Stamper, M. N. Wild, A. Balogh, and G. Jones, Our changing Sun, Astron. Geophys., 40, 4.10-4.16, 1999b.

Mimno, H. R., and P. H. Wang, Continuous Kennelley-Heaviside layer records of a solar eclipse (with suggestions of a corpus- cular effect on Appleton layer). Proc. Inst. Radio Engrs., 21, $529-545,1933$.

Minnis, C. M., Ionospheric behaviour at Khartoum during the eclipse of 25 February, 1952, J. Atmos. Terr. Phys., 6, 91-112, 1954.

Müller-Wodarg, A.-D. Aylward, and M. Lockwood, Effects of a mid-latitude solar eclipse on the thermosphere and ionosphere a modelling study, Geophys. Res. Lett., 25, 3787-3790, 1998.

Rees, M. H., Physics and chemistry of the upper atmosphere, Cambridge University Press, Cambridge, UK, 1989.

Rydbeck, O. E. H., Chalmers solar eclipse ionospheric expedition 1945, Chalm. Tekn. Högsk. Handl., 53, 1946.

Stamper, R., M. Lockwood, M. N. Wild, and T. D. G. Clark, Solar causes of the long-term increase in geomagnetic activity, J. Geophys. Res. in press, 1999. 